\title{
Magnetized particle transport in multi-MA accelerators
}

\author{
N. Bennett๑, ${ }^{1}$ D. R. Welch, ${ }^{2}$ G. Laity, ${ }^{1}$ D. V. Rose $\odot,{ }^{2}$ and M. E. Cuneo ${ }^{1}$ \\ ${ }^{1}$ Sandia National Laboratories, Albuquerque, New Mexico 87185, USA \\ ${ }^{2}$ Voss Scientific, LLC, Albuquerque, New Mexico 87108, USA
}

(Received 19 February 2021; accepted 1 June 2021; published 23 June 2021)

\begin{abstract}
Kinetic simulations of Sandia National Laboratories' $Z$ machine are conducted to understand particle transport in the highly magnetized environment of a multi-MA accelerator. Joule heating leads to the rapid formation of electrode surface plasmas. These plasmas are implicated in reducing accelerator efficiency by diverting current away from the load [M.R. Gomez et al., Phys. Rev. Accel. Beams 20, 010401 (2017), N. Bennett et al., Phys. Rev. Accel. Beams 22, 120401 (2019)]. The fully-relativistic, electromagnetic simulations presented in this paper show that particles emitted in a space-charge-limited manner, in the absence of plasma, are magnetically insulated. However, in the presence of plasma, particles are transported across the magnetic field in spite of being only weakly collisional. The simulated cross-gap currents are well-approximated by the Hall current in the generalized Ohm's law. The Hall conductivities are calculated using the simulated particle densities and energies, and the parameters that increase the Hall current are related to transmission line inductance. Analogous to the generalized Ohm's law, we extend the derivation of the magnetized diffusion coefficients to include the coupling of perpendicular components. These yield a Hall diffusion rate, which is equivalent to the empirical Bohm diffusion.
\end{abstract}

DOI: 10.1103/PhysRevAccelBeams.24.060401

\section{INTRODUCTION}

The pulsed-power TW-class accelerators driving fast $Z$-pinch experiments generate magnetic fields of 100$1000 \mathrm{~T}$ by transporting multi-MA currents to mm-diameter loads $[1,2]$. These high current densities create a charged particle environment in the accelerator transmission lines that is significantly denser $\left(10^{15}-10^{17} \mathrm{~cm}^{-3}[3,4]\right)$ than found in kA-scale accelerators $\left(10^{10}-10^{12} \mathrm{~cm}^{-3}[5-7]\right)$. While kA-class accelerators typically transition from vacuum operation to magnetically insulated transmission lines (MITLs) during the pulse, multi-MA accelerators progress from vacuum to MITL to dense electrode plasma formation within 10 s of $n s[8,9]$. These unique plasma conditions may degrade the accelerator performance by shunting current away from the load.

Surface plasmas form primarily through Joule heating, with particle energy deposition contributing in some locations. These plasmas have the potential to reduce the power coupling to the load at $Z$-pinch facilities such as the $Z$ machine at Sandia National Laboratories [10-12], the Primary Test Stand (PTS) at the China Academy of

Published by the American Physical Society under the terms of the Creative Commons Attribution 4.0 International license. Further distribution of this work must maintain attribution to the author(s) and the published article's title, journal citation, and DOI.
Engineering Physics [13,14], and the MIG generator at the Institute of High Current Electronics [15,16].

The efficiency in current delivery has been studied for the $Z$ machine [17] and the PTS [18] using transmissionline-circuit models. While unable to capture particle motion, these circuit models have increased our understanding of the electrical coupling of the pulse-forming components to the transmission lines and the magnitude of power lost in transit to the load. To understand plasma formation and evolution, particle-in-cell (PIC) methods were advanced to model localized Joule heating and the plasmas resulting from desorbed contaminants in MA-scale transmission lines [19]. It was found that higher rates of cathode plasma desorption decreased the effective transmission line gap and created conditions for power loss.

The PIC electrode-plasma models were further developed [20] and applied more recently to detailed 2D and 3D simulations of the $Z$ machine [9]. These simulations showed that in all but the vacuum stage of operation, some small fraction of the current is shunted across the transmission line gaps. At larger radius in the $Z$ machine, where the current density is a few $10^{4}-10^{5} \mathrm{~A} / \mathrm{cm}^{2}$ and the peak plasma densities are of order $10^{14} \mathrm{~cm}^{-3}$, unmagnetized classical transport accounts for the cross-gap currents. By classical transport, we refer to collisional transport unaided by plasma instabilities. This transport is unmagnetized prior to magnetic insulation or in the vicinity of magnetic nulls $[9,21,22]$. 
At the smallest radii on $Z(r \leq 4.5 \mathrm{~cm})$, where the transmission line approaches the load, the electrode current density exceeds $10^{6} \mathrm{~A} / \mathrm{cm}^{2}$. Here, electrode surface plasmas form during the pulse rise and evolve a Hall-currentrelated conductivity that scales with electron density and enables current to cross the gap in the presence of a strong magnetic field [9].

In this paper, we use kinetic simulations of the $Z$ accelerator to investigate the Hall conductivity in detail and its dominance as the mechanism for cross-gap current at small radii. We stress that any Hall current is not simulated in an ad hoc way, but arises from the selfconsistent calculations of the current densities, charge distributions, and fields. The analytical origin of the Hall conductivity is summarized in Sec. II and compared to a derived Hall-current diffusion rate for a magnetized plasma. We show that this Hall-current diffusion scales with the magnetized diffusion rate by the same factor as the Hall conductivity scales with the conductivity perpendicular to a magnetic field. This Hall-diffusion rate is equivalent to the experimentally derived Bohm diffusion [23].

The classical transport enabled by the Hall conductivity is demonstrated in 2D simulations of a series of $Z$-machine experimental configurations in Sec. V. Although the Hall conductivity is derived in a fluid description that assumes Maxwell distributions, we extract the densities and electric fields from kinetic simulations (with arbitrary distributions) to calculate the analogous cross-gap current. This cross-gap current is also referred to as "current loss" [3]. The experimental configurations modeled include one that was previously presented in Ref. [9] and five related geometries that have different anode-cathode (AK) gap widths but similar initial inductance. Of these, four have a dynamically increasing inductance. These differences enable a further examination of the relationship between load inductance and current loss [3], discussed in Sec. V C.

Details of the simulation particle, field, and surface heating models that capture the plasma dynamics are provided in Sec. III. The $Z$ machine and 3D models of its current-adder region are also described in Sec. III. The 3D models are then used in Sec. IV to show magnetized transport persists at larger radius $(r \geq 5 \mathrm{~cm})$ such that even enhanced space-charged-limited (SCL) emission is largely insulated.

\section{COEFFICIENTS IN MAGNETIZED TRANSPORT}

Particle transport in MA-scale transmission lines occurs despite the presence of very high magnetic fields. Typically, electrode plasmas diffuse across a magnetic field along a density gradient, via interparticle collisions [24]. The conductivity and diffusion coefficient transverse to the magnetic field are reduced by the factor $\left(1+\frac{\omega_{c}^{2}}{\nu_{c}^{2}}\right)^{-1}$ [25], where $\omega_{c}$ is the cyclotron frequency and $\nu_{c}$ is the collision rate. This factor would prohibit cross-gap transport in the inner MITL of the $Z$ accelerator, where magnetic fields exceed $200 \mathrm{~T}$, densities range from $10^{14}-10^{17} \mathrm{~cm}^{-3}$, and $\omega_{c} / \nu_{c}>10^{4}$.

However, experiments and kinetic simulations have shown that current is diverted from the load, especially in the inner MITL [3,9]. This is explained by the Hall current which arises, in a fluid approximation, from the nondiagonal elements of the second-order conductivity tensor [26]. The Hall conductivity couples the current in one direction to electric field components in two directions. Here, we show that a Hall term also exists in the fluid approximation for magnetized diffusion when nondiagonal elements are considered. The Hall diffusion rate, like the Hall conductivity from the generalized Ohm's law [27], is related to unmagnetized transport via $\left(\omega_{c} / \nu_{c}\right) /\left(1+\frac{\omega_{c}^{2}}{\nu_{c}^{2}}\right)$. Thus, transport perpendicular to the magnetic field is feasible, even for $\omega_{c} \gg \nu_{c}$.

The Hall conductivity, $\sigma_{H}$, arises in the generalized Ohm's law presented by Braginskii [27]. The current density, $\mathbf{j}$, is expressed as the sum of components parallel and perpendicular to $\mathbf{B}$ plus a Hall term that establishes a current perpendicular to both $\mathbf{E}^{\prime}$ and $\mathbf{B}$ [28]:

$$
\mathbf{j}=\sigma \mathbf{E}_{\|}^{\prime}+\sigma_{\perp} \mathbf{E}_{\perp}^{\prime}+\sigma_{H}\left(\mathbf{b} \times \mathbf{E}_{\perp}^{\prime}\right) .
$$

Here, $\mathbf{b}=\mathbf{B} / B, \sigma$ is the conductivity parallel to $\mathbf{B}, \sigma_{\perp}$ is perpendicular to $\mathbf{B}$, and $\mathbf{E}^{\prime}$ is the effective electric field in the ion inertial frame:

$$
\mathbf{E}^{\prime}=\mathbf{E}+\left(\mathbf{v}_{i} \times \mathbf{B}\right)+\frac{1}{n_{e} e}\left(\boldsymbol{\nabla} p_{e}-\mathbf{R}_{T}\right),
$$

where $n_{e}$ is the electron density, $p_{e}$ is the electron pressure, $\mathbf{R}_{T}$ is the thermal force [Ref. [27] Eq. (2.9)], and electron inertia and viscosity are ignored. The perpendicular and Hall-term conductivities relate to $\sigma$ by [28]

$$
\begin{aligned}
\sigma & =\frac{n e^{2}}{m \nu_{c}} \\
\sigma_{\perp} & =\frac{\sigma}{1+\frac{\omega_{c}^{2}}{\nu_{c}^{c}}} \quad \text { and }, \\
\sigma_{H} & =\sigma_{\perp} \frac{\omega_{c}}{\nu_{c}}
\end{aligned}
$$

Diffusion from the Hall term is explicit in the diffusion coefficients for magnetized plasmas. To demonstrate, we follow the derivation found in Ref. [25], which begins with the particle flux

$$
\boldsymbol{\Gamma}=\int \mathbf{v} f d \mathbf{v}=-D \boldsymbol{\nabla} n
$$


where $D$ are the diffusion coefficients and the distribution function $f$ may be determined from the Boltzmann equation,

$$
\frac{\partial f}{\partial t}+\mathbf{v} \cdot \frac{\partial f}{\partial \mathbf{r}}+\frac{\mathbf{F}}{m} \cdot \frac{\partial f}{\partial \mathbf{v}}=\left(\frac{\partial f}{\partial t}\right)_{c} .
$$

The collision term in Eq. (3) is assumed a perturbation $[24,25]$ such that

$$
\left(\frac{\partial f}{\partial t}\right)_{c}=-\nu_{c}\left(f-f_{0}\right),
$$

which is solved for $f=f_{0}+f_{1}$, with higher order terms neglected.

For steady-state magnetized transport, Eq. (3) is linearized to

$$
\mathbf{v} \cdot \frac{\partial f_{0}}{\partial \mathbf{r}}+\frac{Z e}{m}(\mathbf{v} \times \mathbf{B}) \cdot \frac{\partial f_{1}}{\partial \mathbf{v}}=-\nu_{c} f_{1} .
$$

where $\mathbf{B}$ is assumed unperturbed. Then assuming $\partial n / \partial y=0, \mathbf{E}=E_{x} \hat{x}, \mathbf{B}=B_{z} \hat{z}$, and an initial Maxwell distribution,

$$
f_{0}=n(\mathbf{r})\left(\frac{m}{2 \pi k_{B} T}\right)^{3 / 2} e^{-m v^{2} / 2 k_{B} T},
$$

and the components of the flux are

$$
\begin{gathered}
\Gamma_{x}=\int\left[-\frac{1}{n \nu_{c}} v_{x} f_{0} v_{x} \frac{\partial n}{\partial x}-\frac{\omega_{c}}{\nu_{c}} v_{x}\left(v_{y}\right) \frac{\partial f_{1}}{\partial v_{x}}\right] d^{3} v \\
=-\frac{k_{B} T}{m \nu_{c}} \frac{\partial n}{\partial x}+\frac{\omega_{c}}{\nu_{c}} \Gamma_{y}, \\
\Gamma_{y}=-\frac{\omega_{c}}{\nu_{c}} \Gamma_{x}, \quad \text { and } \\
\Gamma_{z}=-\frac{k_{B} T}{m \nu_{c}} \frac{\partial n}{\partial z} .
\end{gathered}
$$

Equation (7) is diffusion parallel to $\mathbf{B}$ with coefficient

$$
D_{\|}=\frac{k_{B} T}{m \nu_{c}}=\lambda_{c}^{2} \nu_{c},
$$

where $\lambda_{c}=v_{\text {th }} \nu_{c}$ is the collisional mean free path. Equations (5) and (6) combine to provide diffusion perpendicular to $\mathbf{B}$ :

$$
\begin{aligned}
& \Gamma_{x}=-\frac{k_{B} T}{m \nu_{c}\left(1+\frac{\omega_{c}^{2}}{\nu_{c}^{2}}\right)} \frac{\partial n}{\partial x}, \quad \text { and } \\
& \Gamma_{y}=-\frac{\omega_{c}}{\nu_{c}} \frac{k_{B} T}{m \nu_{c}\left(1+\frac{\omega_{c}^{2}}{\nu_{c}^{2}}\right)} \frac{\partial n}{\partial x}
\end{aligned}
$$

yielding

$$
\begin{aligned}
D_{\perp} & =\frac{k_{B} T}{m \nu_{c}\left(1+\frac{\omega_{c}^{2}}{\nu_{c}^{2}}\right)}=\frac{D_{\|}}{\left(1+\frac{\omega_{c}^{2}}{\nu_{c}^{2}}\right)} \\
& \simeq \frac{m v^{2} \nu_{c}}{m \omega_{c}^{2}} \\
& =r_{L}^{2} \nu_{c},
\end{aligned}
$$

and

$$
\begin{aligned}
D_{H} & =\frac{k_{B} T \omega_{c}}{m \nu_{c}^{2}\left(1+\frac{\omega_{c}^{2}}{\nu_{c}^{2}}\right)}=\frac{\omega_{c}}{\nu_{c}} D_{\perp} \\
\simeq & \frac{k_{B} T}{m \omega_{c}}=\frac{k_{B} T}{e B} \\
& =\frac{m v^{2}}{m \omega_{c}} \\
& =r_{L}^{2} \omega_{c},
\end{aligned}
$$

with the second lines taken in the limit $\omega_{c} / \nu_{c} \gg 1$. Equation (11) is the Bohm diffusion coefficient [23]. The derivation of $D_{\perp}$ has long existed, but we have been unable to locate a derivation that includes Eq. (9). It is possible that the connection between magnetized diffusion, the empirical Bohm diffusion, and the Hall current has not been made previously [29-31]. The relationship between Bohm diffusion and fast electric field fluctuations has been identified [32], and is consistent with the results presented here and in Ref. [9].

The length scale for Eq. (10) is the Larmor radius $\left(r_{L}\right)$ on the collision time scale. The length scale for Bohm diffusion is $r_{L}$ on the cyclotron time scale. This faster rate is comparable to the kinetic solution of the plasma expansion velocities, shown in Sec. VA.

\section{KINETIC MODEL OF THE $Z$ TRANSMISSION LINES}

In the $Z$ machine, 36 pulsed-power modules are connected in parallel to four radial MITLs, labeled "outer MITLs" in Fig. 1. Currents from the outer MITLs are combined within a double post-hole convolute and transferred to a single radial "inner" MITL to deliver up to $26 \mathrm{MA}$ in $100 \mathrm{~ns}$ to the load.

The post-hole convolute electrically connects the anode plates of the four outer MITLs through 12 azimuthally arrayed posts that pass through holes in the cathode plates. A cross section of this geometry is illustrated in Fig. 1. The $Z$ machine fields two convolute variants, one with the posts arrayed at a diameter of $15-\mathrm{cm}$ diameter $(\varnothing 15 \mathrm{~cm})$, illustrated in Fig. 1, and one with a 31-cm diameter $(\varnothing 31 \mathrm{~cm})$.

The inner MITL is tailored for specific loads on $Z$. The inner MITLs and loads modeled in this paper are 


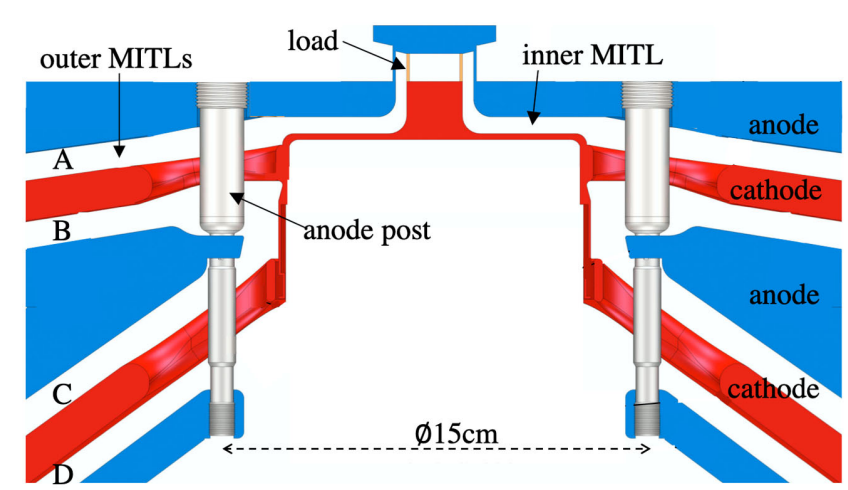

FIG. 1. A cross section of the $Z$ machine outer MITLs, $\varnothing 15 \mathrm{~cm}$ double post-hole convolute (current adder), and inner MITL [33].

specifically for power flow study. Each has an axial transmission line segment at $r=1 \mathrm{~cm}$ that is subject to the highest current densities and rapid surface plasma formation.

To study the generation and evolution of electrode plasmas, simulations of the $Z$ machine convolute and various inner MITL configurations are conducted using the fully-relativistic electromagnetic PIC code CHICAGO [20,34-38]. Simulations of both convolutes are conducted in 3D cylindrical coordinates $(r, \theta, z)$, spanning $15^{\circ}$ azimuthally with symmetry boundaries at $\theta=0^{\circ}$ and $15^{\circ}$ and $100 \mu \mathrm{m}$ minimum cell size. This grid size resolves the dynamics in the surface plasmas for densities less than $10^{17} \mathrm{~cm}^{-3}$ [39], and is sufficient for the $10^{14} \mathrm{~cm}^{-3}$ peak densities in the convolute but not for densities near the load. The plasma sheaths must be resolved for particles to sample the correct local field gradients and generate the correct density gradients. The simulation geometries, shown in Fig. 2, include the final $8 \mathrm{~cm}$ of the outer MITL, the double post-hole convolute, the inner MITL, and load region.

Because no 3D effects on transport are noted in the 3D simulations, 2D simulations are used for high-resolution studies of particle transport $(\Delta r, z=25-50 \mu \mathrm{m})$, using the same field solution and particle treatments as in 3D. This reduces the computation time for grids that resolve the plasma dynamics for densities below $10^{19} \mathrm{~cm}^{-3}$ [39]. The average number of particles per cell is 100 , which was tested to be sufficient for simulation convergence. Current distributions derived from the 3D simulations are injected through the boundaries in $2 \mathrm{D}(r, z)$ simulations of additional inner MITL configurations, in the azimuthally symmetric region interior to the convolute.

The circuit model components of Ref. [17] provide a realistic $Z$ driving pulse. Four voltage pulses are supplied to a BERTHA [40] circuit originating at the water convolute, just outside the insulator stack. In 3D models, the circuit is connected at four grid boundaries to ideal radial or axial feed sections to minimize longitudinal electric fields that are not modeled in the 1D circuits.
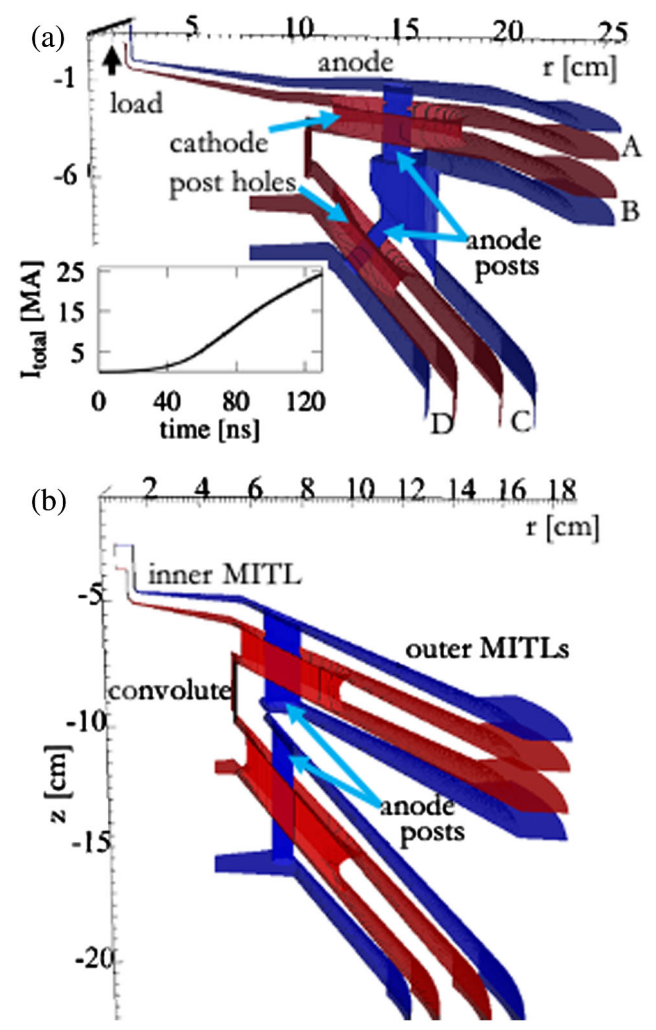

FIG. 2. Illustration of the outer MITLs, double post-hole convolute, and inner MITL fielded for (a) a $\varnothing 31 \mathrm{~cm}$ convolute and (b) a $\varnothing 15 \mathrm{~cm}$ convolute. A pulse-generating circuit is attached at the four outer MITL feeds labeled A, B, C, and D. The total current is inlaid in (a).

For dynamic loads, an imploding-liner impedance is modeled using a second circuit [41].

The simulations use a magnetic-implicit electromagnetic field solver to advance the particles and fields, which relaxes the time step constraint due to cyclotron motion. Reference [42] tabulates allowable values of $\omega_{c} \Delta t \leq 30$ depending in the cyclotron radius and strength of the magnetic field gradient. The simulations presented here use $\omega_{c} \Delta t \leq 2$. An energy-conserving cloud-in-cell model is used to minimize electromagnetic fluctuations from individual macroparticles [43] and a time-biasing algorithm [44] is used to control the growth of electromagnetic fluctuations on the grid.

The electrode surfaces are continuously updated for local temperature increases from Joule heating and particle energy deposition. The Joule heating model derives from [45]

$$
T_{J}(t)=\frac{1}{c_{v}} \int_{0}^{t} \frac{j^{2}(t)}{\sigma} d t
$$

where $\sigma$ and $c_{v}$ are the conductivity and specific heat of the electrode material (assumed constant) and the lineal current density is determined from the curl of the magnetic 
field: $j(t)=\partial H(x, t) / \partial x$, where $H=B / \mu_{0}$ and $x$ is the coordinate normal to the conducting surface cell. Assuming a linearly rising $j(t)$ and that magnetic field diffusion is the dominant contributor over heat conduction, the local temperature increase is

$$
\Delta T_{J}(t) \approx \frac{\vartheta \mu_{0} H^{2}(t)}{2 c_{v}},
$$

which is Eq. (5.2-30) from Ref. [45]. The surface energy factor $\vartheta=1.273$ (from Table 5.2-II of Ref. [45]) and $c_{v}=$ $3.9 \mathrm{~J} / \mathrm{cm}^{3} \mathrm{~K}$ for stainless steel [46,47]. As noted in Ref. [45], the assumption of constant $\sigma$ underestimates the temperature rise but is valid for small $\delta T / T$.

Particle energy deposition $(d E / d s)$ is calculated using the Bethe-Bloch equations for electron and ion energy loss [48]. The path length in a grid cell is $d s=d x / \cos \theta$, where $d x$ is the cell depth and $\theta$ is the particle angle of incidence. The temperature increase $\left(T_{d}(t)\right)$ per macroparticle is then calculated as

$$
\Delta T_{d}=\frac{d E}{d s} \frac{q}{e c_{v} A},
$$

where $q$ is the macroparticle charge, $e$ is the electron charge, and $A$ is the cell's surface area.

The assumption of constant $c_{v}$ in Eqs. (12) and (13) overestimates the temperature rise because $c_{v}$ increases $35 \%$ from 700 to $1727 \mathrm{~K}$ for stainless steel [46]. The sensitivity to the parameters used in Eqs. (12) and (13) were tested in Ref. [9] using simulations in which the desorption rate was varied by a factor of 6 . In this range, there was no change in the plasma transport.

Joule heating melts the electrodes within a $4-\mathrm{cm}$ radius prior to the z-pinch implosion (Fig. 8 of Ref. [9]). The impact of melt on the conductivity and the surface contaminant constituency and inventory, with potential vaporization near the imploding liner, is the focus of a future investigation. The impact on MITL inductance of neglecting melt is estimated using magnetohydrodynamic simulations [49]. A $Z$-relevant model of electrodes carrying 25 MA from $r=0.3$ to $8.0 \mathrm{~cm}$ resulted in electrode expansion within $1 \%$ for $r \geq 2 \mathrm{~cm}$ and $3 \%$ at $r=1 \mathrm{~cm}$.

\section{A. Particle emission models}

Particle emission is modeled as both field and thermal emission of charged particles from the electrode surfaces and thermal desorption of neutral plasma. Electrons are emitted from the cathode after the local electric field stress exceeds the tolerance of the conductor [50,51]. The emission threshold depends on the material and its preparation, with typical values ranging from 150 to $280 \mathrm{kV} / \mathrm{cm}$ as determined when the anode current exceeds the cathode boundary current [5,6]. A previously tested value of $240 \mathrm{kV} / \mathrm{cm}$ [19] is used here. Protons are emitted from the anode after the local surface temperature increases by $400{ }^{\circ} \mathrm{C}$ [52]. The emission rate for these processes is governed by the SCL current density, the nonrelativistic version of which is [50]:

$$
j_{\mathrm{CL}}=\frac{4 \epsilon_{0}}{9}\left(\frac{2 Z e}{m}\right)^{1 / 2} \frac{V_{0}^{3 / 2}}{d^{2}},
$$

where $V_{0}$ is the voltage drop, $d$ is the AK gap width, $m$ is the particle mass, and $Z$ is its charge state. An exact relativistic solution was derived in Ref. [53], the series expansion of which may be written

$$
j_{\text {rel CL }}=\frac{2 m c^{3} \epsilon_{0}}{Z e d^{2}}\left[\left(\frac{Z e V}{m c^{2}}+1\right)^{1 / 2}-0.8471\right]^{2} .
$$

Both the high electric field stress near the cathode and the high temperatures of both electrodes lead to the creation of local surface plasmas. To model plasma formation, a neutral atom or molecule is desorbed from an electrode surface and immediately fragmented to its atomic constituents and ionized, as in Refs. [54,55], and [9]. Thermal ionization [56] is assumed. Previous measurements at Sandia have identified hydrogen, oxygen, and carbon as the primary electrode surface contaminants in the SABRE accelerator [57] and as negative ions in prototypes of the Particle Beam Fusion Accelerator [58]. More recent spectral measurements on the $Z$ machine identify hydrogen as the primary plasma constituent [3]. While carbon was included in models in Ref. [55], hydrogen was the dominant contributor to bipolar currents based on mass, as expressed in Eq. (14) and consistent with the conclusions of Ref. [3]. To reduce the computation time by reducing the number of tracked species, we use hydrogen to represent light ions and oxygen to represent heavy ions. The cross section for $\mathrm{O}^{2+}$ ranges from 1 to 2 orders of magnitude less than the first ionization [59], so higher states are neglected. The excitation process is significant at electron energies below $\sim 40 \mathrm{eV}$, but ionization dominates above this energy so these processes are also neglected.

The $\mathrm{H}_{2} \mathrm{O}$ molecule and its constituents are tracked as kinetic species. The molecule is fragmented and subsequently ionized according to: $\mathrm{H}_{2} \mathrm{O} \rightarrow 2 \mathrm{H}^{+}+\mathrm{O}^{+}+3 e$. The Arrhenius equation calculates the thermal desorption rate as a function of the surface density of adsorbed contaminant particles $(n(t))$ :

$$
\frac{d n(t)}{d t}=-\nu_{t h} n(t) e^{-E^{\prime}(n) /\left(k_{B} T(t)\right)},
$$

where $\nu_{\text {th }} \sim 10^{13} \mathrm{~s}^{-1}, E^{\prime}(n)$ is the effective binding energy, and $T$ is the local surface temperature $[57,60]$. The surface density is measured in monolayers, $n=f n_{M L}$, where $n_{M L} \equiv 10^{15}$ particles $/ \mathrm{cm}^{2}$ and $f$ is a surface roughness factor [61] $(f \sim 8$ for average machining of stainless 
steel [62]). The Temkin isotherm model has been shown to capture the surface-density dependence of $E^{\prime}(n)$ seen in molecular dynamics simulations of $\mathrm{Fe}_{2} \mathrm{O}_{3}$ lattices [63]:

$$
E^{\prime}(n)=E_{d}\left(1-\alpha \frac{n(t)}{f n_{\mathrm{ML}}}\right)[\mathrm{eV}],
$$

where $E_{d}$ is the binding energy at infinitesimal coverage, $\alpha$ is determined experimentally. For the values used, $E_{d}=$ $1 \mathrm{eV}$ is consistent with molecular dynamics calculations and $\alpha=0.17$ is retained from comparisons of simulated currents to data [9] and to a physics-based transmissionline-circuit model of $\mathrm{Z}$ [17]. The initial contaminant inventory on the electrode surfaces, $n(0)$, is taken from the surface roughness factor noted above to be $8.3 \mathrm{ML}$. There is no significance to the decimal place. The simulated current loss has been shown to be insensitive to values between 8 and 16 ML [39].

\section{B. Particle interactions}

The electrons, ions, and neutrals are all governed by kinetic particle algorithms. Thus, the particles are advanced via their individual momenta, collision frequencies, and interaction cross sections, with no assumed distributions. The ionization of neutral particles is expected to occur rapidly due to high electric field stresses, temperatures, and impact ionization. The electron-ion pairs created in ionization events are added to their respective kinetic species. Ion-neutral collisions are also handled in binary fashion assuming a hard-sphere collision model.

Charged particle collisions use a binary Coulomb collision model described in Refs. [64] and [65], in which Nanbu's theory of the cumulative property of Coulomb collisions is applied. Individual particles located within the same grid cell are paired and a Monte-Carlo algorithm determines the effective cumulative Coulomb collision scattering angle for the pair. The correct local velocity distribution is sampled over successive time steps.

Recent work indicates transport coefficients are modified in strong magnetic fields [66]. However, the change in stopping powers in unmagnetized and strongly magnetized regimes is $\sim 25 \%$, and is computationally expensive to extract [67], so modifications to the collision operator are deferred to future work [68].

\section{ENHANCED ION TRANSPORT IN TESLA-SCALE MAGNETIC FIELDS}

An enhanced ion current has been proposed as a possible current loss mechanism in the inner MITL where the electron flow is adjacent to the anode [17,69]. The $\mathbf{E} \times \mathbf{B}$ vectors in the convolute and entrance to the inner MITL create an unusual spatial distribution of flow current in which the electron and ion sheaths are adjacent to the upper anode plate entering the inner MITL [9,70].
This configuration could give rise to a negative charge distribution near the anode that decreases the local electric field and enables more ion SCL emission (for surfaces exceeding the emission threshold).

An enhancement to the Eq. (14) current is analyzed in Ref. [69], but is limited to a 1D electrostatic solution that neglects the applied electric field and the magnetic force on the ions. To quantify a possible enhanced ion emission in the more realistic models presented in this paper, we determine the change in the electric field near the anode surface due to space charge and its impact on Eq. (14).

The expected current densities from Eqs. (14) and (15) for a 7-mm AK gap $[r \sim 5 \mathrm{~cm}$ in Fig. 2(a) $]$ at 0.5-2 MV are plotted in Fig. 3. Simulated electron current densities are also shown. For more direct comparison to Child-Langmuir theory, the electrode plasmas are not modeled in the simulation results in Fig. 3 only. The comparison does include electron and ion emission with accompanying magnetic insulation and vortices in the flow.

An example of the change in the electric field due to the space charge of the electron and ion sheaths is given in Fig. 4(a), which includes electrode plasmas. This example looks at the entrance to the inner MITL $(r=10 \mathrm{~cm})$ for the geometry in Fig. 2(a). In vacuum, the electric fields in the transmission line gaps are flat and the voltages increase linearly from cathode to anode. With SCL emission, the electric field perpendicular to the electrodes $\left(E_{\perp}\right)$ is

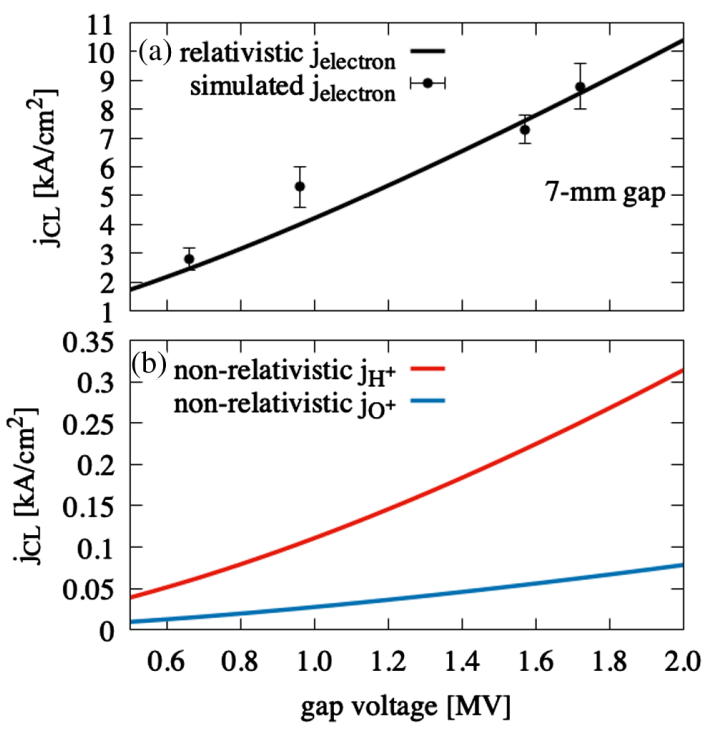

FIG. 3. Child-Langmuir current densities for (a) electrons and (b) $\mathrm{H}^{+}$, and $\mathrm{O}^{+} \cdot j_{\mathrm{H}^{+}}$and $j_{\mathrm{O}^{+}}$are calculated using Eq. (14) for a 7-mm AK gap, and range from $39.3-314 \mathrm{~A} / \mathrm{cm}^{2}$ and $9.86-$ $78.9 \mathrm{~A} / \mathrm{cm}^{2}$, respectively. $j_{\text {electron }}$ is calculated using Eq. (15) and compared to an inner MITL simulation with only field-stress electron emission and thermal $\mathrm{H}^{+}$emission. The simulated $j_{\text {electron }}$ fluctuates due to charge discretization, so an unweighted sliding-average smooth is applied. Error bars indicate the variation in the average value as the width of the sliding average is varied by $1.3 \mathrm{~ns}$. 

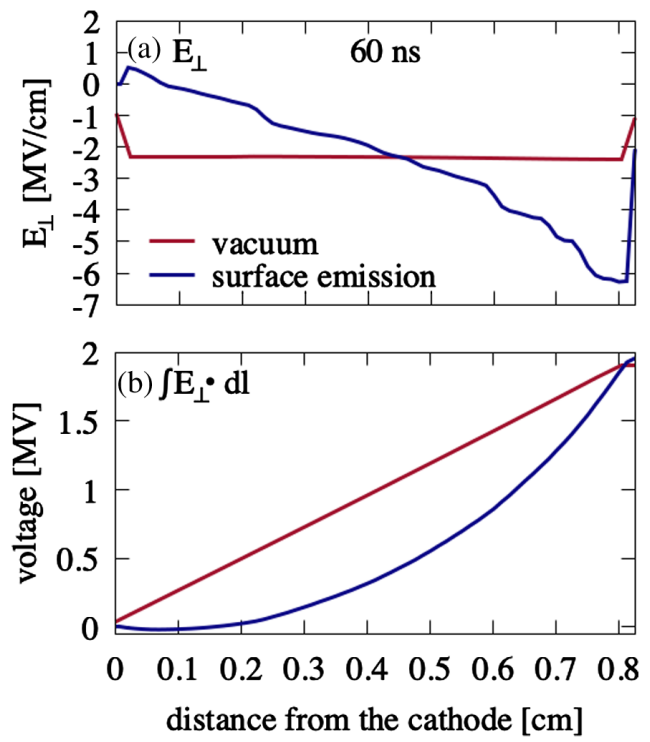

FIG. 4. The values of (a) $E_{\perp}$ and (b) voltage as functions of position in the AK gap in the inner MITL $(r=10 \mathrm{~cm})$ for the simulation geometry in Fig. 2(a). The results without particles are shown in red and results with electrode plasma formation are shown in blue. The values are extracted during the pulse rise [60 ns in the current pulse shown in Fig. 2(a)].

neutralized near the cathode and the voltage increase from cathode to anode appears as a parabola, as in Fig. 2 of Ref. [50]. This is the shape of the voltage in Fig. 4(b) in the case of surface emission with electrode plasmas. A notable difference is the change of sign of $E_{\perp}$ near the cathode due to positive ion charge.

To use $E_{\perp}$ from Fig. 4 to estimate the enhancement to the ion current in Eq. (14), we determine an effective gap width, $d_{\text {eff }}=V_{0} / E_{\perp}$ using $E_{\perp}$ near the anode. At $60 \mathrm{~ns}$ into the current pulse shown in Fig. 2(a), $d_{\text {eff }} \sim d / 3$ leading to a factor of 9 enhancement. Applying this to $\mathrm{H}^{+}$at 1.9 $\mathrm{MV}$ in a $8-\mathrm{mm}$ gap, where $j_{\mathrm{CL}}=223 \mathrm{~A} / \mathrm{cm}^{2}$, the enhancement could lead to over $600 \mathrm{kA}$ of loss interior to the $\varnothing 31 \mathrm{~cm}$ convolute [71]. However, three physical processes mitigate this effect: magnetic insulation, cathode plasma formation, and their space-charge impact on $E_{\perp}$.

As this system evolves, the ions emitted from the anode become less likely to cross the gap due to increasing magnetic insulation and to changes in the charge distribution, to which these ions contribute. The latter is demonstrated in Fig. 5 which compares $E_{\perp}(r=10 \mathrm{~cm})$ at $60 \mathrm{~ns}$ to values at 80 and $100 \mathrm{~ns}$. At $60 \mathrm{~ns}, E_{\perp}$ is created by an electron density imbalance $\left(n_{e}-n_{i}\right)$ near the anode of less than $5 \times 10^{12} \mathrm{~cm}^{-3}$. By $80 \mathrm{~ns}$, this imbalance increases near the anode, however the opposite is occurring near the cathode. In the electrode plasmas near the cathode, $n_{i}>n_{e}$, indicating emitted ions decelerate mid-gap. By $100 \mathrm{~ns}$, the gap is more neutralized.

As the $E_{\perp}$ profile is changing, the ions are becoming more magnetically insulated. To estimate when and where

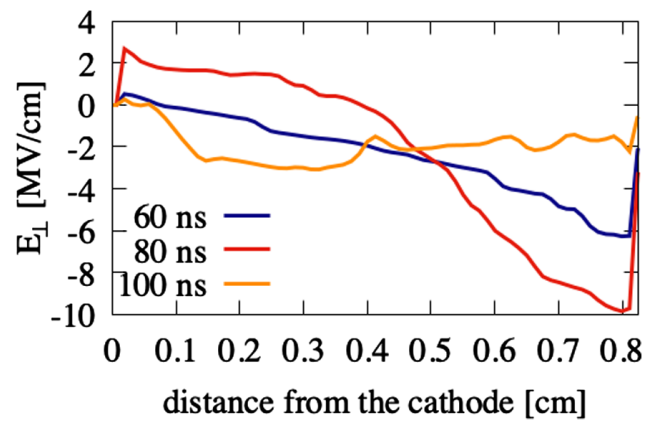

FIG. 5. $E_{\perp}$ as a function of position in the AK gap at 60, 80, and $100 \mathrm{~ns} . E_{\perp}$ is extracted at $r=10 \mathrm{~cm}$ in the simulation geometry in Fig. 2(a).

ions are able to cross the AK gap and contribute to current loss (without Hall-like transport), we estimate the MITL radius below which the ions are magnetically insulated. Using the current pulse in Fig. 2(a) to determine $B_{\theta}(r, t)$ and the voltage $[V(t)]$ at $r=5 \mathrm{~cm}$ to estimate the nonrelativistic ion velocity, the ion Larmor radius equals the gap width $\left(r_{L}=d\right)$ at the MITL radius

$$
r_{\max }=\frac{e}{m} \frac{\mu_{0} I(t)}{2 \pi}\left(\frac{m}{2 Z e V(t)}\right)^{1 / 2} d .
$$

This is plotted in Fig. 6 for $\mathrm{H}^{+}$and $\mathrm{O}^{+}$for a 7-mm gap. Also plotted is the maximum radius for thermal emission from Eq. (12). This shows that for $\mathrm{H}^{+}$, as with electrons, the conditions for insulation exist prior to emission. Although $\mathrm{O}^{+}$is not insulated at emission, the surface area over which $\mathrm{O}^{+}$current loss may occur reduces in time as $j_{\mathrm{CL}}$ is increasing. Its estimated contribution to current loss is tens of kA. Particle energy deposition may increase the emission radius above the dashed line in Fig. 6, but the resulting ions are uninsulated only between 65 and $75 \mathrm{~ns}$ for the simulations presented here.

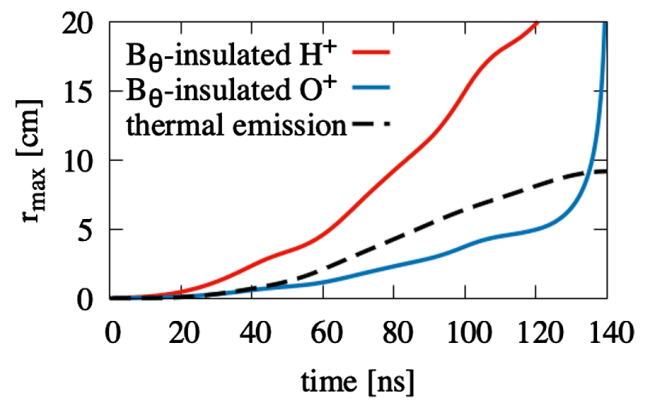

FIG. 6. The maximum radius at which ions are magnetically insulated as a function of time (Eq. (17) for a 7-mm gap. The water constituents $\mathrm{H}^{+}$and $\mathrm{O}^{+}$are plotted in red and blue, respectively. The maximum radius for thermal emission from Eq. (12) for $\Delta T=400 \mathrm{~K}$ (in black) shows thermally emitted $\mathrm{H}^{+}$ are insulated. 
The magnetic insulation feeds back to $E_{\perp}$. As emitted ions become confined to flow near the anode, they are neutralizing the current sheath entering from the convolute. This is consistent with the $E_{\perp}$ profile at $100 \mathrm{~ns}$ in Fig. 5.

While ions emitted from upstream surfaces may not cross the AK gap initially, they do $\mathbf{E} \times \mathbf{B}$ drift downstream into the inner MITL and contribute to the local plasma density there. In this way, they still contribute to current loss, as described in Sec. V.

\section{MAGNETIZED TRANSPORT AT $>10$ TELSA}

The intense magnetic fields in multi-MA accelerators generate electron Larmor radii that are measured in microns. Particles from surface emission are magnetically confined near the electrode surfaces, as briefly described in Sec. IV, and cross-field transport is typically considered for collisional populations $\left(\nu_{c}>\omega_{c}\right)$.

However, collisional transport is not significant in the inner MITL of the $Z$ machine, where the electrode plasmas and the sheath currents entering from the convolute are hot $\left(10^{2}-10^{5} \mathrm{eV}\right)$ and of moderate density $\left(10^{15}-10^{17} \mathrm{~cm}^{-3}\right)$ [9], such that $\nu_{c}<10^{8} \mathrm{~s}^{-1}$. Reference [9] demonstrated that cross-field transport is well-approximated by the Hall conductivity. In this reference, kinetic simulations of the inner MITLs of the $Z$ machine were used to crudely estimate an effective conductivity using a single value of $E_{\mathrm{rms}}$ and the current loss $\left(I_{\text {loss }}\right)$. Discrete values of this effective conductivity showed the same scaling with density as $\sigma_{\mathrm{H}}$.

A more rigorous comparison to $\sigma_{\mathrm{H}}$ is presented here using six inner MITL geometries that differ by length and/or minimum AK gap width. A time-dependent value of $\sigma_{\mathrm{H}}$ is calculated from Eq. (1) for each simulation using $\mathbf{B}(t), n_{e}(t)$, and $\nu_{c}(t)$. For $\nu_{c}(t)$, we use the Coulomb collision rate given by Spitzer for species $\alpha$ scattering off species $\beta$ [72]:

$$
\nu_{\alpha \beta}=\frac{4 \sqrt{2 \pi} e^{4} Z_{\alpha}^{2} Z_{\beta}^{2} n_{\beta} \ln \Lambda_{\alpha \beta}}{\left(4 \pi \epsilon_{0}\right)^{2} 3 m_{\alpha} \mu_{\alpha \beta}}\left(\frac{T_{\alpha}}{m_{\alpha}}+\frac{T_{\beta}}{m_{\beta}}\right)^{-3 / 2},
$$

where $\mu_{\alpha \beta}=m_{\alpha} m_{\beta} /\left(m_{\alpha}+m_{\beta}\right)$ and $\ln \Lambda_{\alpha \beta}$ is the Coulomb logarithm. We describe here how the different MITL geometries result in different $\sigma_{H}(t), \mathbf{E}(t)$, and $I_{\text {loss }}(t)$, but how in all cases the scaling of $I_{\text {loss }}(t)$ is wellapproximated by the Hall current.

Two of the simulation geometries were presented in Ref. [9]. They are fielded on the $\varnothing 31 \mathrm{~cm}$ convolute and differ by minimum AK gap width, as illustrated in Fig. 7. The electron density contour maps at $100 \mathrm{~ns}$ are also shown in the figure. The height of the final coaxial segment is adjusted to give the two geometries similar integrated inductances. The same is true for the four additional inner-MITL simulations. These will be fielded on the $\varnothing 15 \mathrm{~cm}$ convolute [Fig. 2(b)] and so terminate at a smaller

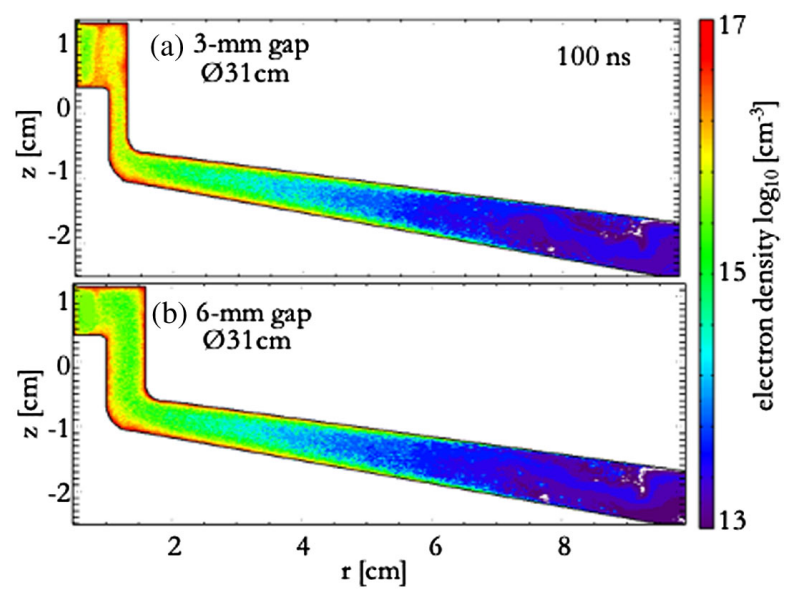

FIG. 7. 2D simulation geometries for the two $\varnothing 31 \mathrm{~cm}-$ convolute inner MITLs identified by their AK gap widths at $z=0$ of (a) $3 \mathrm{~mm}$ and (b) $6 \mathrm{~mm}$. The electron density contours are also plotted in each figure at $100 \mathrm{~ns}$.

radius. Their geometries are illustrated in Fig. 8 along with the electron density contours and $B_{\theta}$ isosurfaces at $60 \mathrm{~ns}$.

The timings of the electron density contours in Figs. 7 and 8 are chosen to illustrate two key developments as the

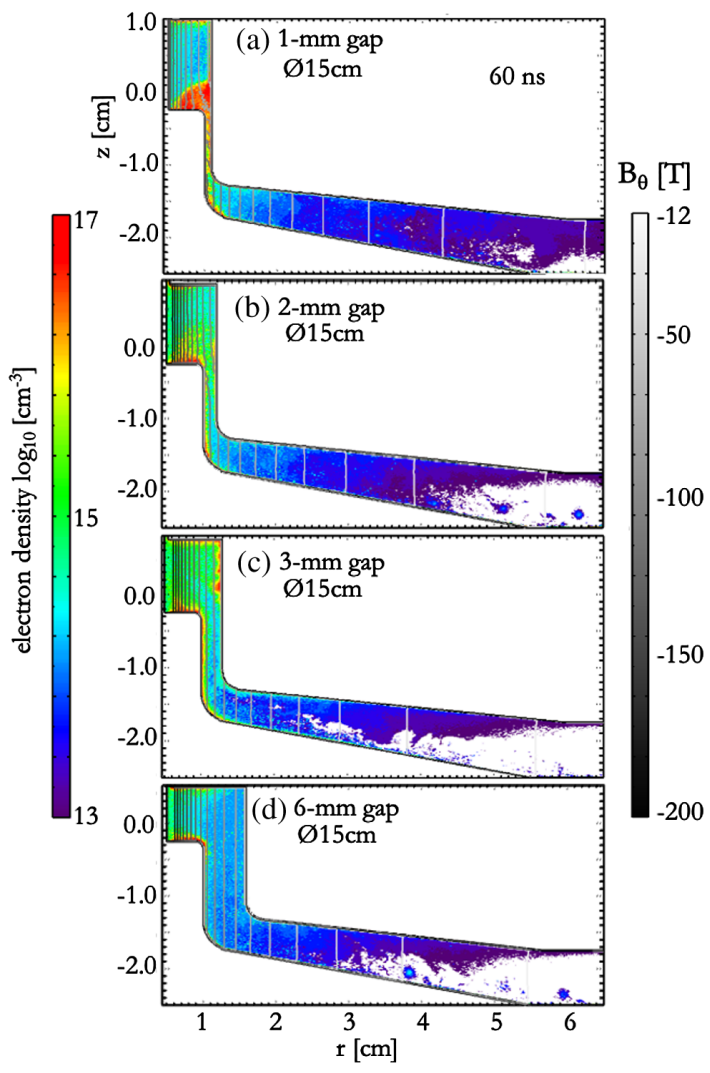

FIG. 8. 2D simulation geometries for the four $\varnothing 15 \mathrm{~cm}-$ convolute inner MITLs identified by their AK gaps at $r=z=$ $-1 \mathrm{~cm}$ of (a) $1 \mathrm{~mm}$, (b) $2 \mathrm{~mm}$, (c) $3 \mathrm{~mm}$, and (d) $6 \mathrm{~mm}$. The electron density contours and magnetic field isosurfaces are also plotted in each figure at $60 \mathrm{~ns}$. 
AK gap width is reduced (keeping $r_{C}$ fixed). The contaminant inventory is proportional to the surface area, so the total inventories, and the densities near the electrodes, are similar in all cases. This inventory flows downstream into a volume that decreases with AK gap width, so the local density increases with decreasing (local) gap. From Fig. 8, $B_{\theta} \geq 100 \mathrm{~T}$ while $n_{e} \sim 10^{15}-10^{17} \mathrm{~cm}^{-3}$, so $\omega_{c} \gg \nu_{c}$ and $\sigma_{\mathrm{H}} \sim n_{e} e^{2} / m \omega_{c}$. The linear relationship between $\sigma_{\mathrm{H}}$ and $n_{e}$ leads to the first development, which is that geometries that increase density generate larger cross-gap transport.

The second key development is that as the cross-gap current density increases, the $\mathbf{j} \times \mathbf{B}$ force may change the density distribution. This occurs for the configurations in Fig. 8(a) and (b) (1 and 2-mm gaps, $\varnothing 15 \mathrm{~cm}$ convolute). Figure 8(a) shows the time during which the density exceeds $10^{17} \mathrm{~cm}^{-3}$ across the gap over an axial length of a few $\mathrm{mm}$. The $\mathbf{j} \times \mathbf{B}$ force on this slice of plasma sweeps it downstream and opens the gap back up. While the total particle charge is similar for all configurations, the plasma occupies a larger volume for the larger gaps and does not carry enough $\mathbf{j}$ to sweep downstream.

As reported in Ref. [9], the current loss occurs along a transmission line length of a few $\mathrm{cm}$. The plasma accumulated in this region is a combination of locally desorbed particles and sheath currents generated at larger radius that drift downstream. The electrode plasma expansion velocity presented in Sec. VA is too slow for the surface plasmas alone to fill the AK gap above a few $10^{14} \mathrm{~cm}^{-3}$. However, the combined surface and sheath distributions, reflected in Figs. 7 and 8, may contain sufficient density that current loss may be accounted for by the Hall conductivity, as detailed in Sec. V B. The structures that increase the local $\mathbf{E}$ field and densities are related to inductance in Sec. V C, as is appropriate for these inductively loaded MITLs.

\section{A. Electrode plasma expansion}

We roughly estimate the plasma expansion velocity $\left(v_{\text {plasma }}\right)$ as the rate at which an ion $10^{16}-\mathrm{cm}^{-3}$ density contour migrates into the AK gap. The average $v_{\text {plasma }}$ is calculated from the cathode near $r=1 \mathrm{~cm}$ for each $\varnothing 15 \mathrm{~cm}$-convolute simulation in Fig. 8. For the 1-mm through 6-mm gaps, the $v_{\text {plasma }}$ estimates are $1.3,1.6,0.98$, and $0.92 \mathrm{~cm} / \mu \mathrm{s}$, respectively. (The average $v_{\text {plasma }}$ obtained using a $10^{15}-\mathrm{cm}^{-3}$ density contour is $1.6 \mathrm{~cm} / \mu \mathrm{s}$ for the 2-mm and 6-mm gaps. The inflow of particles from upstream complicates estimates using density contours $<10^{15}-\mathrm{cm}^{-3}$.)

To determine if the simulated plasma expansion is consistent with the diffusion rate in Eq. (11), we estimate $D_{H}$ using the global values of $T_{i}(t)$ and $B_{\theta}(t)$ for each simulation. The estimates for $D_{H}$ are plotted in Fig. 9, where the average $v_{\text {plasma }}$ is included on a separate axis. To draw a comparison, Eq. (2) may be rewritten $D_{H} \nabla n=n \mathbf{v}$. Then, for surface densities of order $10^{16} \mathrm{~cm}^{-3}$ and minimum gradients of order $10^{17} \mathrm{~cm}^{-4}$ (from Figs. 7 and 8),

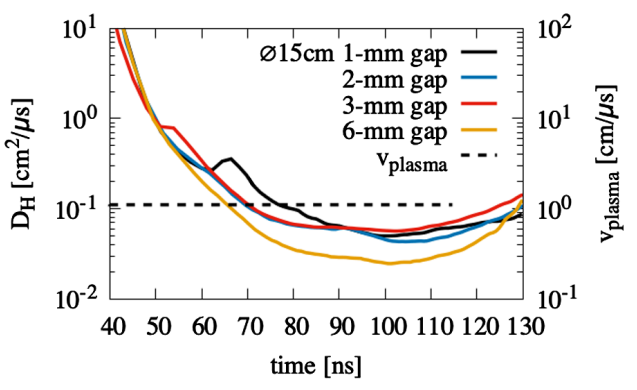

FIG. 9. $D_{H}$ from Eq. (11) estimated for the Fig. 8 geometries. Using $D_{H} \nabla n=n \mathbf{v}$, with $n \sim 10^{16} \mathrm{~cm}^{-3}$ and $\nabla n \sim 10^{17} \mathrm{~cm}^{-4}$ from simulation, $D_{H}$ provides an estimated $v$ that is consistent with the simulated average plasma expansion velocity shown on the right axis.

$D_{H}$ provides $1 \mathrm{D}$ expansion velocities that are of the same order as $v_{\text {plasma }}$ in the $2 \mathrm{D}$ simulations. In this rough comparison, Hall physics is not inconsistent with the simulated plasma expansion, and it is suitable to consider its role in current loss.

\section{B. Cross-gap current}

The densities in the gap from expanding electrode plasmas are supplemented by particles flowing downstream. The resulting plasmas span the $\mathrm{AK}$ gaps with minimum densities exceeding $10^{15} \mathrm{~cm}^{-3}$ out to $r \sim 2-3 \mathrm{~cm}$, as seen in Figs. 7 and 8.

The regions of plasma build-up correspond to the locations of cross-gap current, as illustrated in Figs. 10, 11,12 , and 13. These figures map the values of $r B_{\theta}$ (net current) versus position. These are negative for the way we have oriented the MITLs. Two contour plots are shown for each of the geometries in Fig. 8; they represent the current distributions at $50 \mathrm{~ns}$ ( $23.5 \mathrm{MA}$ into the pulse) and $100 \mathrm{~ns}$ ( 18 MA). These distributions indicate that the majority of current loss is generally distributed along a $1-5 \mathrm{~cm}$ segment of the MITL and not confined to a single arc. This is supportive of the $r B_{\theta}$ map reported in Ref. [9] for the Fig. 8(a) geometry.

The exceptions to this distributed current loss occur early in the simulations with the smaller gaps, Figs. 10(a) and 11(a). In these two cases, a plasma density exceeding $10^{17} \mathrm{~cm}^{-3}$, along a length of a few mm, carries most of the loss current. This high current density generates a nonnegligible $j \times B$ acceleration toward the load. While this is occurring, the plasma density is still increasing in the inner MITL, so the loss current fluctuates without dropping to zero.

The fluctuations in current loss for the smaller AK gaps are seen in Fig. 14. The current loss is calculated as the difference between the anode currents recorded at outer radius and near the load $\left(I_{\text {loss }}=I_{\text {in }}-I_{\text {load }}\right)$. The timedependent $I_{\text {loss }}(t)$ is compared to a Hall current calculated using $\sigma_{\mathrm{H}}(t) E(t) A$. Here, $E$ is the electric field from 

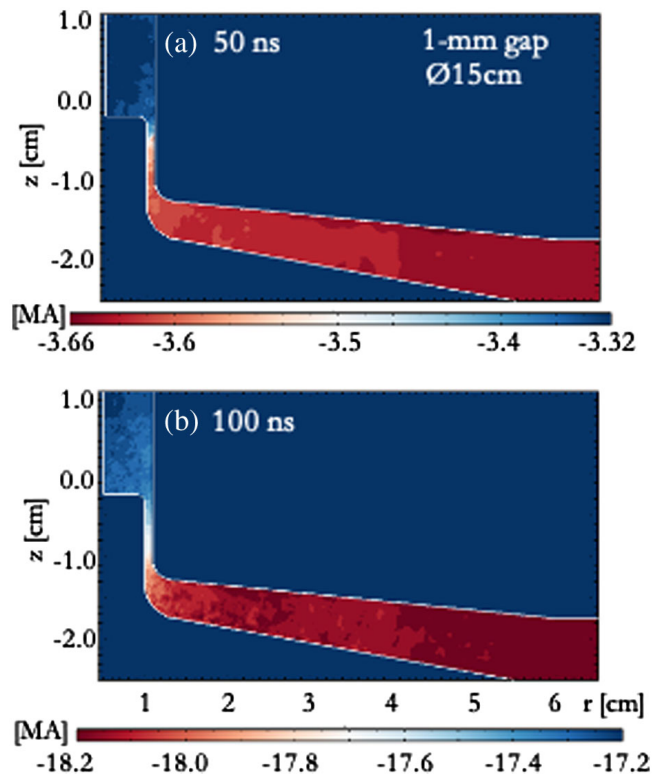

FIG. 10. Current distribution ( $r B_{\theta}$ map) in the $\varnothing 15 \mathrm{~cm} 1-\mathrm{mm}$ gap simulation [Fig. 8(a)] at (a) $50 \mathrm{~ns}$ and (b) $100 \mathrm{~ns}$.

$V_{\text {gap }}(t) / d$ at $r=1 \mathrm{~cm}$ and $A$ is the anode surface area to $r=3 \mathrm{~cm}$. (A fixed value of $\mathrm{A}$ is used with the timedependent parameters. The value of $r=3 \mathrm{~cm}$ is chosen crudely as the universal extent of the $10^{15} \mathrm{~cm}^{-3}$ minimum densities.) $\sigma_{H}$ is calculated from Eq. (1) using $B_{\theta}(t)$, the average $n_{e}(t)$ in the gap (excluding the electrode surfaces) to $r=3 \mathrm{~cm}$, and $\nu_{c}(t)$ from the average $T_{e}(t)$.

This volume-averaged Hall current calculation, from time-history probes in the simulation, fails to capture the $I_{\text {loss }}$ fluctuations in Figs. 14(d) and (c) near 60 and 80 ns, respectively. These large, transient increases in $I_{\text {loss }}$ are a
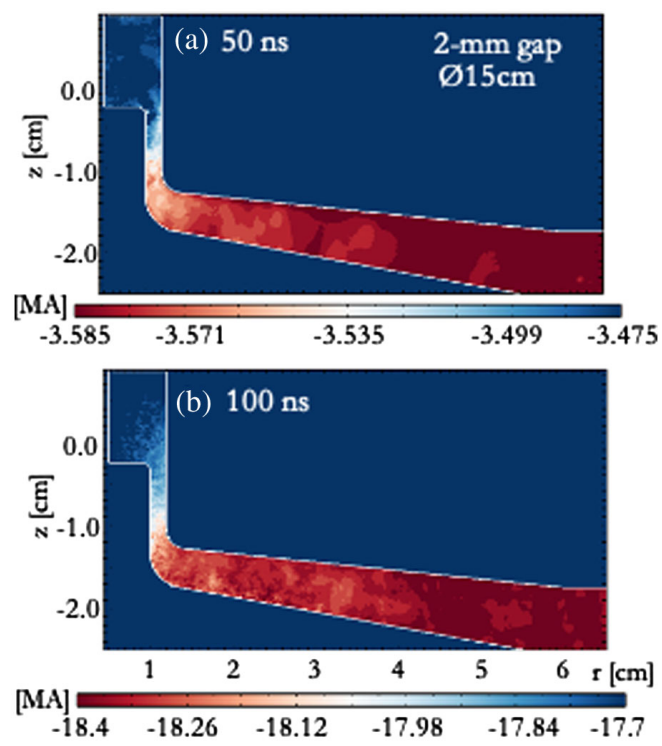

FIG. 11. Current distribution in the $\varnothing 15 \mathrm{~cm} \mathrm{2-mm} \mathrm{gap} \mathrm{sim-}$ ulation [Fig. 8(b)] at (a) $50 \mathrm{~ns}$ and (b) $100 \mathrm{~ns}$.

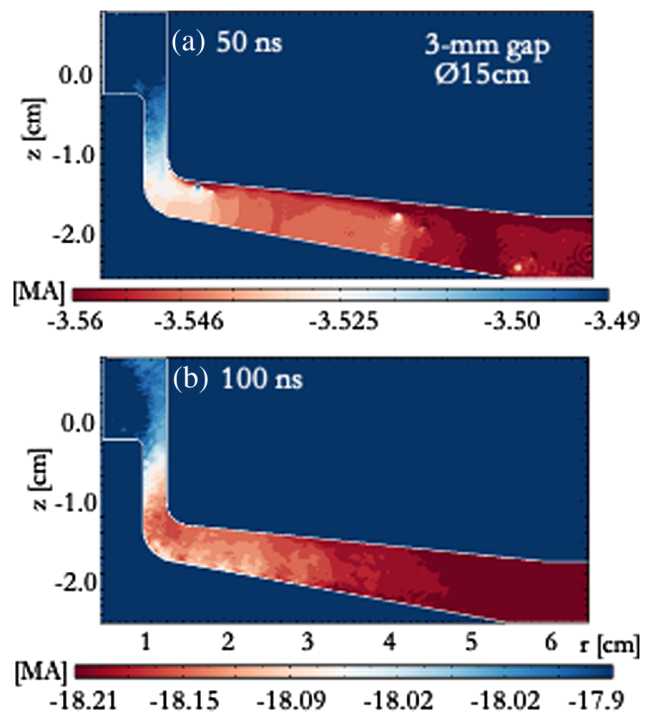

FIG. 12. Current distribution in the $\varnothing 15 \mathrm{~cm} 3$-mm gap simulation [Fig. 8(c)] at (a) $50 \mathrm{~ns}$ and (b) $100 \mathrm{~ns}$.

result of the large $\mathbf{j}$ and subsequent $\mathbf{j} \times \mathbf{B}$ motion in the narrow, higher-density plasma regions. These are alluded to in the changing current distributions in Figs. 10 and 11. We more accurately estimate $\sigma_{H} E A$ in the $1.0<r<1.2 \mathrm{~cm}$ region using $2 \mathrm{D}$ contours of $n_{e}$ and $E$ generated as snapshots in a simulation. This yields the discrete values of the Hall current, calculated for 40, 50, and $60 \mathrm{~ns}$, that are plotted in Figs. 14(c) and (d). Because $n_{e}$ and $E$ are drawn from smoothed 2D contour plots, error bars are included.

The Hall current is generally in good agreement with $I_{\text {loss }}(t)$ for the four geometries in Fig. 14. The volumeaveraged Hall current is calculated for the Fig. 7 geometries and plotted in Fig. 15. The agreement provides evidence that transport perpendicular to strong magnetic fields in

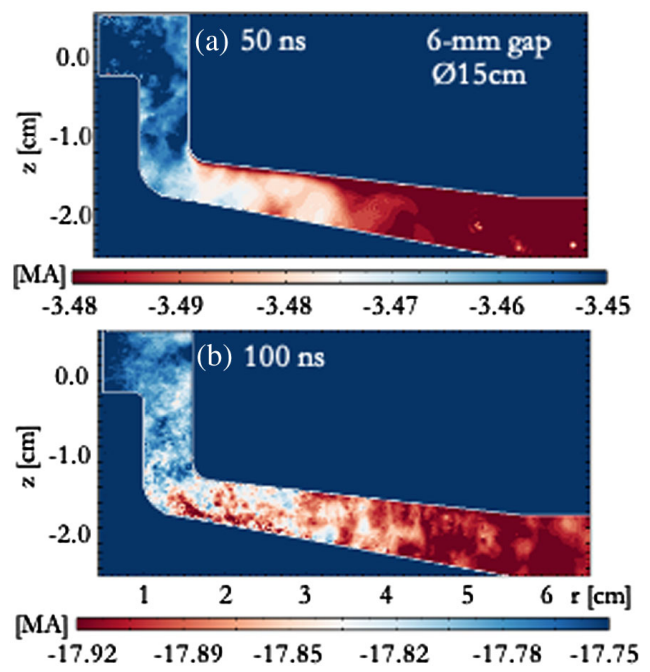

FIG. 13. Current distribution in the $\varnothing 15 \mathrm{~cm} 6-\mathrm{mm}$ gap simulation [Fig. 8(d)] at (a) $50 \mathrm{~ns}$ and (b) $100 \mathrm{~ns}$. 


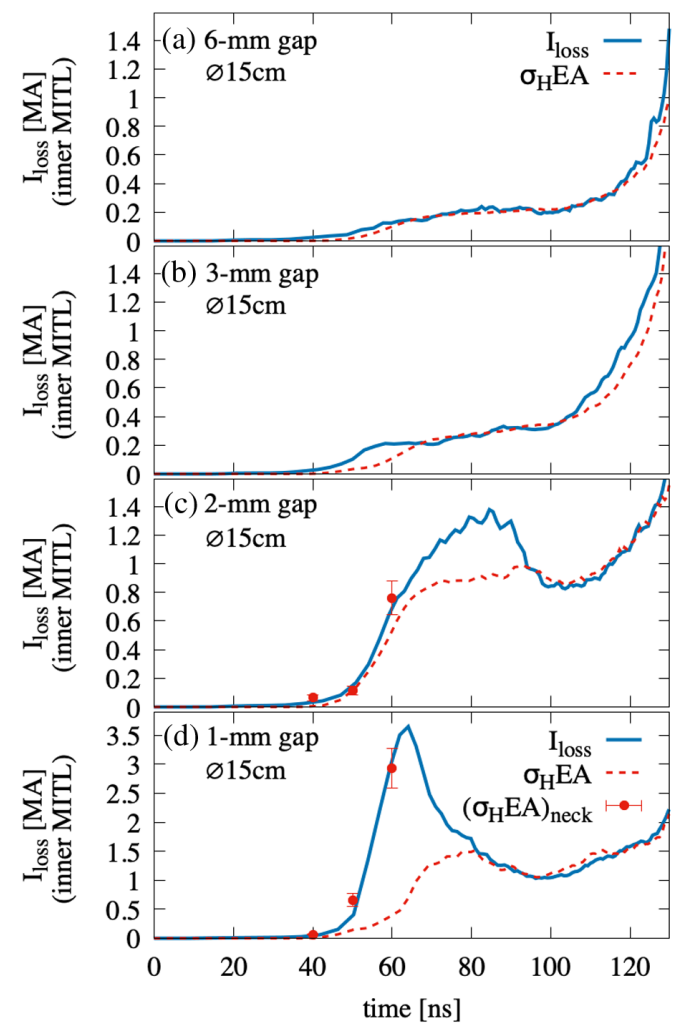

FIG. 14. Current loss $\left(I_{\text {loss }}=I_{\text {in }}-I_{\text {load }}\right)$ compared to an estimate of the Hall current $\left(\sigma_{H} E A\right)$ for the four geometries fielded on the $\varnothing 15 \mathrm{~cm}$ convolute (Fig. 8). The volume-average Hall current, given by the dotted lines, has an estimated error of $11 \%$ from using $V_{\text {gap }}(t)$ from a single location and average $n_{e}(t)$, both of which fluctuate at the $8 \%$ level over $0.5 \mathrm{~mm}$. The points in (c) and (d) are calculated from local values in the minimum AK gap region, averaged over a $7-\mathrm{mm}$ axial region. The mean of the resulting radial distribution is used with statistical errors defined as the $10 \%-90 \%$ values.

these inner MITLs is a result of the Hall terms. Experimental campaigns and diagnostics for $\mathrm{Z}$ are in development to further test this result.

\section{Role of inductance}

Figure 14 shows the average $I_{\text {loss }}$ increases as the minimum AK gap width decreases, as expected based on upstream plasma accumulating in smaller volumes. In addition, there is a dramatic increase in $I_{\text {loss }}$ for all gaps at $\sim 110$ ns. This latter increase is not caused by density but by an increase in $\mathbf{E}$ that begins at that time. A dynamic load is attached to these $\varnothing 15 \mathrm{~cm}$-convolute inner MITLs to mimic the change in inductance from an imploding $Z$-pinch liner. The same liner mass is used in each, even though the axial extents of the loads are different for each, as illustrated in Fig. 8. The induced $E_{\perp}$ is increased by more than $50 \%$ during implosion.

There is a different cause for the more gradual increase in $I_{\text {loss }}$ seen in Fig. 15. The loads on the two $\varnothing 31 \mathrm{~cm}$-convolute

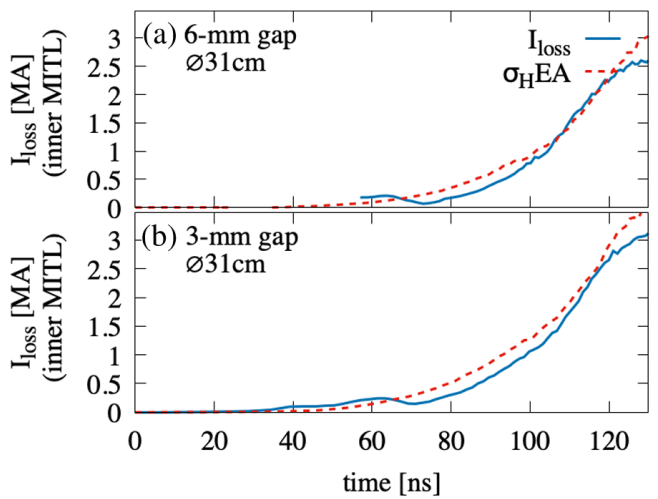

FIG. 15. Current loss $\left(I_{\text {loss }}=I_{\text {in }}-I_{\text {load }}\right)$ compared to an estimate of the Hall current $\left(\sigma_{H} E A\right)$ for (a) the 6-mm gap and (b) the 3-mm gap fielded on the $\varnothing 31 \mathrm{~cm}$ convolute (Fig. 7). The volume-average Hall current has an estimated $11 \%$ error as described in Fig. 14.

inner MITLs are static, but the MITLs are longer, with more surface area for contaminant desorption. The result is more plasma available to accumulate in the downstream region. Using Fig. 7(a) and Fig. 8(c) as examples, the minimum gap widths are the same but the densities at $100 \mathrm{~ns}$ are $\sim 8 \times$ larger for the longer MITL, which is approximately their difference in upstream surface area.

This provides some explanation of the relationship between load inductance and $I_{\text {loss }}$ that was introduced in Ref. [3]. The inner MITL carries the full accelerator current, so Joule heating and particle energy deposition increase most surface temperatures above the desorption threshold. Thus a longer MITL, with higher total inductance, generates more plasma that contributes to $\sigma_{H}$ downstream. This is true for the longer radial segment on the $\varnothing 31 \mathrm{~cm}$ convolute and for longer coaxial sections on either convolute. This adds to the impact of increases in the $\mathbf{E}$ from a time-dependent increase in the load inductance.

The total inductance is not the sole determining factor, however. Where and how the inductance increases also matters. For simulations in which the AK gap is increased at small radius, the total inductance increases but the $E_{\perp}$ and density are reduced, thus reducing $I_{\text {loss }}$.

\section{SUMMARY}

Kinetic simulations of the $Z$ machine have been conducted in $3 \mathrm{D}$ and $2 \mathrm{D}$ to understand particle transport in the highly magnetized environment of a multi-MA accelerator. While the transmission lines in kA-scale accelerators are dominated by SCL electron emission, at the MA scale, joule heating and particle energy deposition lead to the rapid formation of electrode surface plasmas. These plasmas may ultimately degrade the accelerator performance by shunting current away from the load.

The 3D simulation was conducted of the $Z$ machine's current adder region. Results show that because electrons 
and ions generated in the adder region drift downstream into the inner MITL along the anode surface, their spacecharge enhancements to the electric field can increase SCL ion emission from the anode. This effect is mitigated as protons emitted from the anode are magnetically insulated, thereby neutralizing any local space-charge imbalance. The magnetic insulation of the emitted ions, and the restoring effect they have on the local electric field, precludes them from significantly enhancing current loss via unmagnetized classical transport.

The enhanced ion emission does, however, increase the plasma density downstream in the inner MITL. This plasma density is a factor in the Hall conductivity that is derived in the generalized Ohm's law [27,28]. In an analogous treatment, we extend the derivation of the magnetized diffusion coefficients [25] to include the coupling of perpendicular components. In this way, we derive an analogous Hall diffusion rate, which is the empirical Bohm diffusion [23].

The 2D simulations resolve the dynamics in the inner MITLs. Comparisons of six inner MITL geometries show that the time-dependent current loss (the difference between the currents at the outer radius and the load) is well approximated by a Hall current calculated by $\sigma_{H} E A$. We stress that no Hall terms need be added to the fully-kinetic simulations. The Hall effect arises from the self-consistent calculations of the current densities, charge distributions, and fields. Instabilities may contribute to transport, but are not analyzed.

Because the current losses are Hall-driven, they are limited by the plasma densities and local $\mathbf{E}$, rather than by a space-charge-limited or magnetically-insulated current loss mechanism. Thus, the current loss may be mitigated by reducing the contaminant inventory and the induced $\mathbf{E}$.

It is in this way that the inner MITL inductance is related to the current loss, as has been observed experimentally [3]. Longer transmission lines that increase the contaminant inventory, and hence the electrode plasma density, also increase the integrated inductance. The Hall current is linearly proportional to the induced electric fields, as well. Where and how the inductance increases matters, though. If the AK gap is increased at small radius, the total inductance increases but the density and E are reduced.

A complex analysis of the breakdown of the desorbed contaminants is underway. This study incorporates electron and ion impact ionization, dissociative ionization, and attachment for desorbed water molecules. The goal is a prediction of the plasma ionization fraction for a range of system energy densities. Further studies will include the roles of photoionization and radiation transport.

Finally, code validation is on-going. The $3 \mathrm{D}$ and $2 \mathrm{D}$ geometries in Figs. 2(a) and 7(a) are compared to measurements in Ref. [9]. The geometries in Fig. 8 will be compared to data in a future work.

\section{ACKNOWLEDGMENTS}

Sandia National Laboratories is a multi-mission laboratory managed and operated by National Technology \& Engineering Solutions of Sandia, LLC., a wholly owned subsidiary of Honeywell International, Inc., for the U.S. Department of Energy's National Nuclear Security Administration under Contract No. DE-NA0003525. The views expressed in the article do not necessarily represent the views of the U.S. Department of Energy or the United States Government. This project was partially supported by LDRD Project No. 209240.

[1] M. K. Matzen, Phys. Plasmas 4, 1519 (1997).

[2] M. G. Haines, Plasma Phys. Controlled Fusion 53, 093001 (2011).

[3] M. R. Gomez, R. M. Gilgenbach, M. E. Cuneo, C. A. Jennings, R. D. McBride, E. M. Waisman, B. T. Hutsel, W. A. Stygar, D. V. Rose, and Y. Maron, Phys. Rev. Accel. Beams 20, 010401 (2017).

[4] A. Porwitzky, D. H. Dolan, M. R. Martin, G. Laity, R. W. Lemke, and T. R. Mattsson, Phys. Plasmas 25, 063110 (2018).

[5] N. Bruner, T. Genoni, E. Madrid, D. Rose, D. Welch, K. Hahn, J. Leckbee, S. Portillo, B. Oliver, V. Bailey, and D. Johnson, Phys. Rev. ST Accel. Beams 11, 040401 (2008).

[6] N. Bennett et al., Phys. Rev. ST Accel. Beams 17, 050401 (2014).

[7] A. S. Richardson, J. C. Zier, J. T. Engelbrecht, S. B. Swanekamp, J. W. Schumer, D. Mosher, P. F. Ottinger, D. L. Duke, T. J. Haines, M. P. McCumber, and A. Gehring, Phys. Rev. Accel. Beams 22, 050401 (2019).

[8] D. R. Welch, N. Bennett, D. Rose, C. Thoma, and W. Stygar, Phys. Rev. Accel. Beams 22, 070401 (2019).

[9] N. Bennett, D. R. Welch, C. A. Jennings, E. Yu, M. H. Hess, B. T. Hutsel, G. Laity, J. K. Moore, D. V. Rose, K. Peterson, and M. E. Cuneo, Phys. Rev. Accel. Beams 22, 120401 (2019).

[10] R. B. Spielman et al., Phys. Plasmas 5, 2105 (1998).

[11] M. E. Savage, K. R. LeChien, M. R. Lopez, B. S. Stoltzfus, W. A. Stygar, D. S. Artery, J. A. Lott, and P. A. Corcoran, in 2011 IEEE Pulsed Power Conference (2011), pp. 983-990, https://doi.org/10.1109/PPC.2011.6191629.

[12] D. B. Sinars et al., Phys. Plasmas 27, 070501 (2020).

[13] D. Jianjun, X. Weiping, F. Suping, W. Meng, L. Hongtao, S. Shengyi, X. Minghe, H. An, T. Qing, G. Yuanchao, G. Yongchao, W. Bin, Z. Wenkang, H. Xianbin, W. Lijuan, Z. Zhaohui, H. Yi, and Y. Libing, IEEE Trans. Plasma Sci. 41, 2580 (2013).

[14] J. Deng et al., Matter Radiat. Extremes 1, 48 (2016).

[15] B. A. Kablambaev, A. V. Luchinsky, V. K. Petin, N. A. Ratakhin, S. A. Sorokin, V. F. Fedushchak, S. A. Chaikovsky, and S. V. Shlyakhtun, Vopr. At. Nauki Tekhn. 34, 149 (2001).

[16] S. A. Sorokin, Phys. Plasmas 23, 043110 (2016).

[17] B. T. Hutsel, P. A. Corcoran, M. E. Cuneo, M. R. Gomez, M. H. Hess, D. D. Hinshelwood, C. A. Jennings, G. R. Laity, D. C. Lamppa, R. D. McBride, J. K. Moore, 
A. Myers, D. V. Rose, S. A. Slutz, W. A. Stygar, E. M. Waisman, D. R. Welch, and B. A. Whitney, Phys. Rev. Accel. Beams 21, 030401 (2018).

[18] W. Zou, F. Guo, L. Chen, S. Song, M. Wang, W. Xie, and J. Deng, Phys. Rev. ST Accel. Beams 17, 110401 (2014).

[19] D. V. Rose, E. A. Madrid, D. R. Welch, R. E. Clark, C. B. Mostrom, W. A. Stygar, and M. E. Cuneo, Phys. Rev. ST Accel. Beams 18, 030402 (2015).

[20] D. R. Welch, N. Bennett, T. C. Genoni, C. Thoma, and D. V. Rose, Phys. Rev. Accel. Beams 23, 110401 (2020).

[21] T. D. Pointon, W. A. Stygar, R. B. Spielman, H. C. Ives, and K. W. Struve, Phys. Plasmas 8, 4534 (2001).

[22] D. V. Rose, D. R. Welch, T. P. Hughes, R. E. Clark, and W. A. Stygar, Phys. Rev. ST Accel. Beams 11, 060401 (2008).

[23] D. Bohm, The Characteristics of Electrical Discharges in Magnetic Fields, edited by A. Guthrie and R. Wakerling (McGraw-Hill, New York, 1949), Chap. 2.

[24] M. N. Rosenbluth and A. N. Kaufman, Phys. Rev. 109, 1 (1958).

[25] T. Boyd and J. Sanderson, The Physics of Plasmas (Cambridge University Press, Cambridge, England, 2003).

[26] R. Balescu, Transport Processes in Plasmas (North Holland, Amsterdam, 1988).

[27] S. I. Braginskii, in Reviews of Plasma Physics, Vol. 1, edited by M. A. Leontovich (Consultants Bureau, New York, 1965), p. 205.

[28] B. V. Somov, Plasma Astrophysics: Part I Fundamentals and Practice (Springer Science+Business Media, LLC, New York, 2006).

[29] L. Spitzer, Phys. Fluids 3, 659 (1960).

[30] J. B. Taylor and B. McNamara, Phys. Fluids 14, 1492 (1971).

[31] H. R. Kaufman, J. Vac. Sci. Technol. B 8, 107 (1990).

[32] E. Bultinck, S. Mahieu, D. Depla, and A. Bogaerts, J. Phys. D 43, 292001 (2010).

[33] W. A. Stygar et al., Phys. Rev. ST Accel. Beams 12, 120401 (2009).

[34] D. Welch, D. Rose, B. Oliver, and R. Clark, Nucl. Instrum. Methods Phys. Res., Sect. A 464, 134 (2001), proc. of the 13th Int. Symp. on Heavy Ion Intertial Fusion.

[35] D. R. Welch, D. V. Rose, R. E. Clark, T. C. Genoni, and T. P. Hughes, Comp. Phys. Comm. 164, 183 (2004), proceedings of the 18th International Conference on the Numerical Simulation of Plasmas.

[36] D. Rose, D. Welch, B. Oliver, R. Clark, D. Johnson, J. Maenchen, P. Menge, C. Olson, and D. Rovang, J. Appl. Phys. 91, 3328 (2002).

[37] D. Welch, T. Genoni, R. Clark, and D. Rose, J. Comput. Phys. 227, 143 (2007).

[38] C. Thoma, D. R. Welch, R. E. Clark, D. V. Rose, and I. E. Golovkin, Phys. Plasmas 24, 062707 (2017).

[39] G. R. Laity et al., Towards predictive plasma science and engineering through revolutionary multiscale algorithms and models, final report, Sandia National Laboratories Tech. Report No. SAND2021-0718, 2021.

[40] D. Hinshelwood, Naval Research Laboratory, Washington, DC, Memo. Report No. 5185, 1983.
[41] M. E. Cuneo et al., IEEE Trans. Plasma Sci. 40, 3222 (2012).

[42] T. C. Genoni, R. E. Clark, and D. R. Welch, Open Plasma Phys. J. 3, 36 (2010).

[43] C. K. Birdsall and A. B. Langdon, Plasma Physics via Computer Simulation (Adam Hilger, New York, 1991).

[44] B. B. Godfrey, Time-biased field solver for electromagnetic codes, Mission Research Corp. Tech. Report No. AMRCN-138, 1980; presented at the 9th Conference on the Numerical Simulation of Plasmas.

[45] H. Knoepfel, Magnetic Fields (John Wiley and Sons, New York, 2000).

[46] J. Valencia and P. Quested, in ASM Handbook, Vol. 15: Castings (ASM International, Materials Park, Ohio, 2008).

[47] W. A. Stygar, S. E. Rosenthal, H. C. Ives, T. C. Wagoner, G. O. Allshouse, K. E. Androlewicz, G. L. Donovan, D. L. Fehl, M. H. Frese, T. L. Gilliland, M. F. Johnson, J. A. Mills, D. B. Reisman, P. G. Reynolds, C. S. Speas, R. B. Spielman, K. W. Struve, A. Toor, and E. M. Waisman, Phys. Rev. ST Accel. Beams 11, 120401 (2008).

[48] W. Leo, Techniques for Nuclear and Particle Physics Experiments, 2nd ed. (Springer-Verlag, New York Berlin Heidelberg, 1994).

[49] K. Cochrane, M. Desjarlais, M. Lane, K. Leung, A. Thompson, and A. Wright, Desorption source modeling: From electronic structure, molecur dynamics, Temkin isotherm, to finite element calculations, Sandia National Laboratories Tech. Report No. SAND2018-8709C, 2018.

[50] I. Langmuir, Phys. Rev. 2, 450 (1913).

[51] R. Fowler and L. Nordheim, Proc. R. Soc. A 119, 173 (1928).

[52] T. W. L. Sanford, J. A. Halbleib, J. W. Poukey, A. L. Pregenzer, R. C. Pate, C. E. Heath, R. Mock, G. A. Mastin, D. C. Ghiglia, T. J. Roemer, P. W. Spence, and G. A. Proulx, J. Appl. Phys. 66, 10 (1989).

[53] H. Jory and A. Trivelpiece, J. Appl. Phys. 40, 3924 (1969).

[54] N. Bruner, D. R. Welch, K. D. Hahn, and B. V. Oliver, Phys. Rev. ST Accel. Beams 14, 024401 (2011).

[55] N. Bennett, D. R. Welch, T. J. Webb, M. G. Mazarakis, M. L. Kiefer, M. D. Crain, D. W. Droemer, R. E. Gignac, M. D. Johnston, J. J. Leckbee, I. Molina, D. Nielsen, R. Obregon, T. Romero, S. Simpson, C. C. Smith, F. L. Wilkins, and D. Ziska, Phys. Plasmas 22, 033113 (2015).

[56] A. A. Noyes and H. A. Wilson, Proc. Natl. Acad. Sci. U.S.A. 8, 303 (1922).

[57] M. E. Cuneo, P. R. Menge, D. Hanson, W. Fowler, M. Bernard, G. Ziska, A. Filuk, T. Pointon, R. Vesey, D. Welch, J.E. Bailey, M. Desjarlais, T. Lockner, T. Mehlhorn, S. Slutz, and M. Stark, IEEE Trans. Plasma Sci. 25, 229 (1997).

[58] J. P. VanDevender, R. W. Stinnett, and R. J. Anderson, Appl. Phys. Lett. 38, 229 (1981).

[59] R. R. Laher and F. R. Gilmore, J. Phys. Chem. Ref. Data 19, 277 (1990).

[60] P. Redhead, The Physical Basis for Ultrahigh Vacuum (Chapman and Hall, London, 1968).

[61] M. Li and H. F. Dylla, J. Vac. Sci. Technol. A 11, 1702 (1993).

[62] H. F. Dylla, D. M. Manos, and P. H. LaMarche, J. Vac. Sci. Technol. A 11, 2623 (1993). 
[63] J. M. D. Lane, K. Leung, A. P. Thompson, and M.E. Cuneo, J. Phys. Condens. Matter 30, 465002 (2018).

[64] K. Nanbu and S. Yonemura, J. Comput. Phys. 145, 639 (1998).

[65] D. P. Higginson, I. Holod, and A. Link, J. Comput. Phys. 413, 109450 (2020).

[66] S. D. Baalrud and J. Daligault, Phys. Rev. Lett. 110, 235001 (2013).

[67] L. Jose and S. D. Baalrud, Phys. Plasmas 27, 112101 (2020).

[68] E. S. Evans, S. A. Cohen, and D. R. Welch, Phys. Plasmas 25, 042105 (2018).
[69] E. M. Waisman, M. P. Desjarlais, and M. E. Cuneo, Phys. Rev. Accel. Beams 22, 030402 (2019).

[70] E. A. Madrid, D. V. Rose, D. R. Welch, R. E. Clark, C. B. Mostrom, W. A. Stygar, M. E. Cuneo, M. R. Gomez, T. P. Hughes, T. D. Pointon, and D. B. Seidel, Phys. Rev. ST Accel. Beams 16, 120401 (2013).

[71] D. V. Rose, E. M. Waisman, M. P. Desjarlais, M. E. Cuneo, B. T. Hutsel, D. R. Welch, N. Bennett, and G. R. Laity, Phys. Rev. Accel. Beams 23, 080401 (2020).

[72] P. W. Rambo and R. J. Procassini, Phys. Plasmas 2, 3130 (1995). 